\title{
Politische Repräsentation von Frauen in den Mitgliedstaaten der Europäischen Union im Vergleich
}

\author{
Beate Hoecker
}

In der ersten Dekade des 21. Jahrhunderts ist Politik nach wie vor eine männliche Domäne. Auch wenn innerhalb der Europäischen Union mit Angela Merkel in Deutschland seit 2005 eine Kanzlerin regiert, Finnland aktuell sowohl ein weibliches Staatsoberhaupt (Tarja Halonen) als auch eine Ministerpräsidentin (Mari Kiviniemi) hat und auch die Slowakei seit kurzem eine Premierministerin (Iveta Radicova) vorweisen kann, sind Frauen in politischen Spitzenpositionen keineswegs eine Selbstverständlichkeit. Weltweit gibt es derzeit lediglich zehn gewählte weibliche Staatsoberhäupter und gleichfalls nur zehn Regierungschefinnen. ${ }^{1}$ Auf der Ebene der nationalen Parlamente erreichen von insgesamt 145 Staaten allein 25 Staaten einen Frauenanteil von über 30 Prozent, wobei aktuell Ruanda den Spitzenplatz einnimmt. ${ }^{2}$ Bemerkenswert ist, dass zu diesen 25 Ländern mit einer so genannten critical mass immerhin sieben Mitgliedstaaten der EU zählen.

Die anhaltende Unterrepräsentation von Frauen in der Politik beschäftigt seit längerem auch die politikwissenschaftliche Frauen- und Geschlechterforschung. Aus theoretischer Sicht liegen zwar durchaus unterschiedliche Begründungsmuster für eine paritätische Vertretung von Frauen und Männern in der Politik vor, besonders stark aber ist das Argument der Geschlechtergleichheit und -gerechtigkeit. ${ }^{3}$ Empirisch hat sich der Forschungsstand zur politischen Repräsentation von Frauen in Europa insbesondere in den letzten zehn Jahren deutlich ausgeweitet. ${ }^{4}$ Und auch auf europäischer Ebene ist seit Mitte der 1990er Jahre die Frage nach der politischen Chancengleichheit von Frauen und Männern zunehmend in den

1 Weibliche Staatsoberhäupter haben zurzeit Argentinien, Costa Rica, Finnland, Indien, Irland, Kirgisistan, Liberia, Litauen, die Schweiz und die Föderation Bosnien und Herzegowina. Eine Regierungschefin gibt es in Australien, Bangladesch, Kroatien, Finnland, Deutschland, Island, der Slowakei, Trinidad und Tobago, den Niederländischen Antillen und den Aland Inseln. Vgl. http://www.guide2womenleaders.com (Abruf am 11. August 2010).

2 Vgl. Inter-Parliamentary Union, http://www.ipu.org/wmn-e/classif.htm, Stand vom 30. Juni 2010 (Abruf am 11. August 2010).

3 Vgl. Anne Phillips, Democracy and Representation: Or, Why Should It Matter Who Our Representatives Are?, in: dies. (Hrsg.), Feminism and Politics, Oxford 1998, S. $224-240$.

4 Für die Anfänge der europaweit vergleichenden genderbezogenen Repräsentationsforschung siehe insbesondere Joni Lovenduski / Pippa Norris (Hrsg.), Gender and Party Politics, London 1993; Beate Hoecker (Hrsg.), Handbuch Politische Partizipation von Frauen in Europa. Die Mitgliedstaaten, Band I, Opladen 1998. Neuere Analysen vorrangig zu West- beziehungsweise Osteuropa liegen unter anderem vor von Mercedes Mateo Diaz, Representing Women? Female Legislators in West European Parliaments, Colchester 2005; Joni Lovenduski (Hrsg.), State Feminism and Political Representation, Cambridge 2006; Beate Hoecker / Gesine Fuchs (Hrsg.), Politische Partizipation von Frauen in Europa. Die Beitrittsstaaten, Band II, Wiesbaden 2004; Yvonne Galligan I Sara Clavero / Marina Calloni, Gender Politics and Democracy in Post-Socialist Europe, Opladen 2007; Marilyn Rueschemeyer / Sharon L. Wolchik (Hrsg.), Women in Power in Post-Communist Parliaments, Washington 2009. Einen Überblick zu allen 27 EU-Staaten gibt Johanna Kantola, Women's Political Representation in the European Union, in: The Journal of Legislative Studies, 15. Jg. (2009), H. 4, S. $379-400$. 
Fokus gerückt. So betont die Europäische Kommission die Bedeutung der Gleichstellung der Geschlechter als Grundrecht und gemeinsamen Wert der EU und sieht in der Unterstützung einer gleichberechtigten Vertretung von Frauen und Männern in Entscheidungspositionen einen vorrangigen Aktionsbereich. ${ }^{5}$ Darüber hinaus gibt es seitens der Kommission inzwischen vermehrt Dokumentationen und Analysen zur politischen Repräsentation von Frauen in den Mitgliedstaaten. ${ }^{6}$ Vor diesem Hintergrund ist es Ziel des Beitrags, einen Überblick zum Stand der deskriptiven politischen Repräsentation von Frauen in den EU-Mitgliedstaaten sowie auf europäischer Ebene zu geben und Erklärungsansätze für die im Ländervergleich insgesamt höchst unterschiedliche weibliche Repräsentation aufzuzeigen. ${ }^{7}$

\section{Statistische Bestandsaufnahme}

\subsection{Die parlamentarische Repräsentation von Frauen in den EU-Staaten}

Parlamente gelten als Schlüsselinstitutionen demokratischer Systeme, und die Struktur ihrer Mitgliedschaft ist nicht zuletzt ein Indikator für politische Chancengleichheit. Aktuell stellen Frauen knapp ein Viertel (24 Prozent) aller Abgeordneten in den nationalen Parlamenten der EU. Von diesem Durchschnittswert gibt es jedoch erhebliche Abweichungen; insgesamt erstreckt sich die Bandbreite der parlamentarischen Frauenanteile von 46 Prozent (Schweden) bis 9 Prozent (Malta) (vgl. Tabelle 1). Unterteilt man die parlamentarische Repräsentation von Frauen in die Kategorien „hoch“ (über 30 Prozent), „mittel“ (20 bis 30 Prozent) und „niedrig“ (unter 20 Prozent), ergeben sich für die 27 Staaten folgende Einordnungen:

Die Spitzengruppe wird dominiert von Schweden (46 Prozent), den Niederlanden (42) und Finnland (40). Auf deutlich über 30 Prozent kommen zudem Dänemark (38), Belgien (38) und Spanien (37), während Deutschland (33) erst mit einigem Abstand folgt. Damit rekrutiert sich die Spitze ausschließlich aus den alten EU-Mitgliedern, wobei neben den

5 Vgl. Europäische Kommission, Ein Fahrplan für die Gleichstellung von Frauen und Männern 2006-2010, Brüssel 2006, S. 2. Im Widerspruch dazu steht allerdings, dass in der 2010 neu gebildeten Kommission Chancengleichheit keinen eigenständigen Verantwortungsbereich mehr darstellt. Der Ungar László Andor ist nunmehr Kommissar für Beschäftigung, Soziales und Integration, zuvor hieß es noch Beschäftigung, Soziales und Chancengleichheit.

6 Siehe Europäische Kommission, Frauen in der europäischen Politik - Zeit zu handeln, Luxemburg 2009; dies., Frauen und Männer in Entscheidungspositionen 2007, Luxemburg 2008. Seit 2004 liegen jährliche Gleichstellungsberichte vor; zuletzt Europäische Kommission, Bericht zur Gleichstellung von Frauen und Männern 2010, Luxemburg 2009. Empirische Daten zur politischen Repräsentation von Frauen auf nationaler wie europäischer Ebene präsentiert die Kommission zudem online in ihrer „Datenbank Frauen und Männer in Entscheidungspositionen“.

7 Das Konzept der deskriptiven Repräsentation geht zurück auf Hanna F. Pitkin und beschreibt die Zusammensetzung einer Vertretungskörperschaft, die sozialstrukturell und demographisch möglichst ein Spiegelbild der Gesellschaft sein soll („standing for"). Davon zu unterscheiden ist nach Pitkin die substanzielle Repräsentation, nach der die Repräsentanten im Interesse der Repräsentierten handeln (,acting for"). Vgl. Hanna F. Pitkin, The Concept of Representation, Berkeley 1967. Zur Kritik daran siehe Suzanne S. Schüttemeyer / Sven T. Siefken, Parlamente in der EU: Gesetzgebung und Repräsentation, in: Oscar W. Gabriel / Sabine Kropp (Hrsg.), Die EU-Staaten im Vergleich. Strukturen, Prozesse, Politikinhalte, Wiesbaden 2008, S. 482 - 513, S. 502 f. 


\begin{tabular}{|c|c|c|c|c|}
\hline \multicolumn{5}{|c|}{ Tabelle 1: Frauen in den nationalen Parlamenten der EU-Staaten 2010} \\
\hline Land & Wahljahr & $\begin{array}{c}\text { Abgeordnete } \\
\text { (gesamt / Frauen) }\end{array}$ & $\begin{array}{l}\text { Frauenanteil } 2010 \\
\text { (in Prozent) }\end{array}$ & $\begin{array}{l}\text { Frauenanteil } 2000 \\
\text { (in Prozent) }\end{array}$ \\
\hline Schweden & 2006 & $349 / 162$ & 46 & 43 \\
\hline Niederlande & 2006 & $150 / 63$ & 42 & 36 \\
\hline Finnland & 2007 & $200 / 80$ & 40 & 37 \\
\hline Dänemark & 2007 & $179 / 68$ & 38 & 37 \\
\hline Belgien & 2007 & $150 / 57$ & 38 & 23 \\
\hline Spanien & 2008 & $350 / 128$ & 37 & 28 \\
\hline Deutschland & 2009 & $622 / 205$ & 33 & 31 \\
\hline Österreich & 2008 & $183 / 51$ & 28 & 27 \\
\hline Portugal & 2009 & $230 / 63$ & 27 & 19 \\
\hline Estland & 2007 & $101 / 23$ & 23 & 18 \\
\hline Lettland & 2006 & $100 / 22$ & 22 & 17 \\
\hline Großbritannien & 2010 & $650 / 143$ & 22 & 18 \\
\hline Tschechien & 2010 & $200 / 44$ & 22 & 15 \\
\hline Bulgarien & 2009 & $240 / 50$ & 21 & 11 \\
\hline Italien & 2008 & $630 / 134$ & 21 & 11 \\
\hline Luxemburg & 2009 & $60 / 12$ & 20 & 17 \\
\hline Polen & 2007 & $460 / 92$ & 20 & 13 \\
\hline Litauen & 2008 & $141 / 27$ & 19 & 18 \\
\hline Frankreich & 2007 & $577 / 109$ & 19 & 11 \\
\hline Slowakei & 2006 & $150 / 27$ & 18 & 14 \\
\hline Griechenland & 2009 & $300 / 52$ & 17 & 9 \\
\hline Irland & 2007 & $165 / 23$ & 14 & 12 \\
\hline Slowenien & 2008 & $90 / 13$ & 14 & 10 \\
\hline Zypern & 2006 & $56 / 7$ & 13 & 7 \\
\hline Rumänien & 2008 & $334 / 38$ & 11 & 7 \\
\hline Ungarn & 2010 & $386 / 35$ & 9 & 8 \\
\hline Malta & 2008 & $69 / 6$ & 9 & 9 \\
\hline \multicolumn{5}{|l|}{ EU-Durchschnitt } \\
\hline EU-27 & & $7122 / 1734$ & 24 & 19 \\
\hline EU-15 & & $4795 / 1350$ & 28 & 22 \\
\hline EU-12 & & $2327 / 384$ & 17 & 12 \\
\hline \multicolumn{5}{|c|}{$\begin{array}{l}\text { Quellen: Europäische Kommission, Datenbank Frauen und Männer in Entscheidungspositionen (Stand } \\
\text { vom 2. März 2010); Vergleichsdaten nach dem Archiv der Inter-Parliamentary Union (Stand vom April } \\
\text { 2000). }\end{array}$} \\
\hline
\end{tabular}

traditionell als frauenfreundlich geltenden nordischen Staaten inzwischen auch Belgien und Spanien den Sprung nach oben geschafft haben.

Das Mittelfeld führen Österreich (28) und Portugal (27) an. Dicht beieinander liegen dann die baltischen Staaten Estland (23) und Lettland (22) sowie Großbritannien (22), Tschechien (22), Bulgarien (21), Italien (21), Luxemburg (20) und Polen (20).

Die 20-Prozent-Marke knapp verpasst haben Litauen (19) und Frankreich (19), gefolgt von der Slowakei (18) und Griechenland (17). Am unteren Ende der Rangordnung stehen Irland (14), Slowenien (14), Zypern (13), Rumänien (11), Ungarn (9) und Malta (9).

Insgesamt kommt somit lediglich ein Drittel der EU-Staaten auf überdurchschnittliche Frauenanteile im nationalen Parlament; zwei Drittel liegen unter dem EU-Durchschnitt von 24 Prozent, wobei sechs Länder nicht einmal 15 Prozent erreichen. In die Rubrik mit unterdurchschnittlichen Frauenanteilen im nationalen Parlament fallen neben sechs Staaten 
der alten EU zudem alle neuen Mitglieder aus Ost- und Mitteleuropa sowie die Mittelmeerinseln Zypern und Malta. ${ }^{8}$ Die seit der Erweiterung im Jahr 2004 bestehende Kluft zwischen den alten und neuen Mitgliedsländern zeigt sich auch bei der getrennten Berechnung der Durchschnittswerte: Während die 15 alten EU-Staaten 2010 im Durchschnitt einen parlamentarischen Frauenanteil von 28 Prozent vorweisen können, erzielen die Beitrittsstaaten hier lediglich 17 Prozent.

Vergleicht man den derzeitigen Stand mit früheren Daten, zeigt sich ein moderater Aufwärtstrend. In den letzten zehn Jahren (2000 bis 2010) stieg der durchschnittliche parlamentarische Frauenanteil in den 15 alten EU-Staaten von 22 auf 28 Prozent, in den neuen von rund 12 auf 17 Prozent. Damit fällt die Steigerungsrate unter den alten Ländern (+ 6 Prozentpunkte) etwas höher aus als unter den Beitrittsländern (+5) und hat zugleich die Kluft zwischen ihnen noch vergrößert. ${ }^{9}$ Im Kreis der alten EU-Staaten konnten insbesondere Belgien (+ 15), Italien (+ 10), Spanien (+ 9) sowie Portugal, Frankreich und Griechenland (jeweils +8 ) ihre parlamentarischen Frauenanteile erkennbar steigern, während dagegen für Deutschland $(+2)$, Irland $(+2)$ sowie Österreich $(+1)$ kaum Fortschritte zu verzeichnen sind. Unter den neuen Ländern fallen allein Bulgarien $(+10)$ und Polen $(+7)$ durch hohe Steigerungsraten auf; nur marginale Veränderungen gab es demgegenüber in Litauen (+ 1), Ungarn (+ 1) und Malta (+ 0 Prozentpunkte).

Angesichts dieser Daten kann nicht von einer „angemessenen“, geschweige denn paritätischen Vertretung von Frauen in den nationalen Parlamenten die Rede sein (abgesehen von wenigen Ausnahmen). Die Vermutung, dass der durch die Europäisierung bedingte Machtverlust der Parlamente zu ihrer verstärkten Feminisierung führen könnte, findet somit - zumindest bislang - keine Bestätigung. ${ }^{10}$ Unter Beibehaltung der jetzigen durchschnittlichen Steigerungsrate würden Frauen vielmehr erst in fünfzig Jahren (2060) europaweit die Hälfte aller Parlamentssitze einnehmen. So viel Langmut bringt die europäische Bevölkerung allerdings nicht auf. Nach einer repräsentativen Umfrage von Eurobarometer aus dem Jahr 2009 sieht mehr als die Hälfte der Befragten (55 Prozent) eine Veränderung der parlamentarischen Unterrepräsentation von Frauen als dringend geboten; unter den Frauen fiel der Zuspruch zudem erkennbar höher aus (61 Prozent) als unter den Männern (49 Prozent). ${ }^{11}$

\subsection{Die Repräsentation von Frauen in den Regierungen der EU-Staaten}

Als Schaltstellen der politischen Macht gelten die Regierungen. Wie Tabelle 2 zeigt, sind Frauen derzeit durchschnittlich zu gut einem Viertel (27 Prozent) in den nationalen Regierungen vertreten; 2005 lag dieser Anteil noch bei einem Fünftel (22 Prozent). ${ }^{12}$ Damit fällt die Regierungsbeteiligung von Frauen aktuell etwas höher aus als ihre parlamentarische

8 Im Jahr 2004 traten Estland, Lettland, Litauen, Polen, Tschechien, Ungarn, Slowenien, die Slowakei sowie Malta und Zypern der EU bei; Bulgarien und Rumänien folgten 2006.

9 Vgl. zum früheren Stand der parlamentarischen Frauenanteile auch Beate Hoecker, a.a.O. (Fn. 4), S. 381 - 383; Beate Hoecker / Gesine Fuchs, Politische Partizipation und Repräsentation von Frauen in den Beitrittsstaaten im Vergleich, in: dies. (Hrsg.), a.a.O. (Fn. 4), S. 285 - 306, S. 287 f.

10 Vgl. zu dieser These ebenda, S. 304.

11 Vgl. Special Eurobarometer 326, Gender Equality in the EU in 2009, Brüssel 2010, S. 95 ff.

12 Angabe für 2005 nach WSI FrauenDatenReport 2005, Berlin 2005, S. 391. 


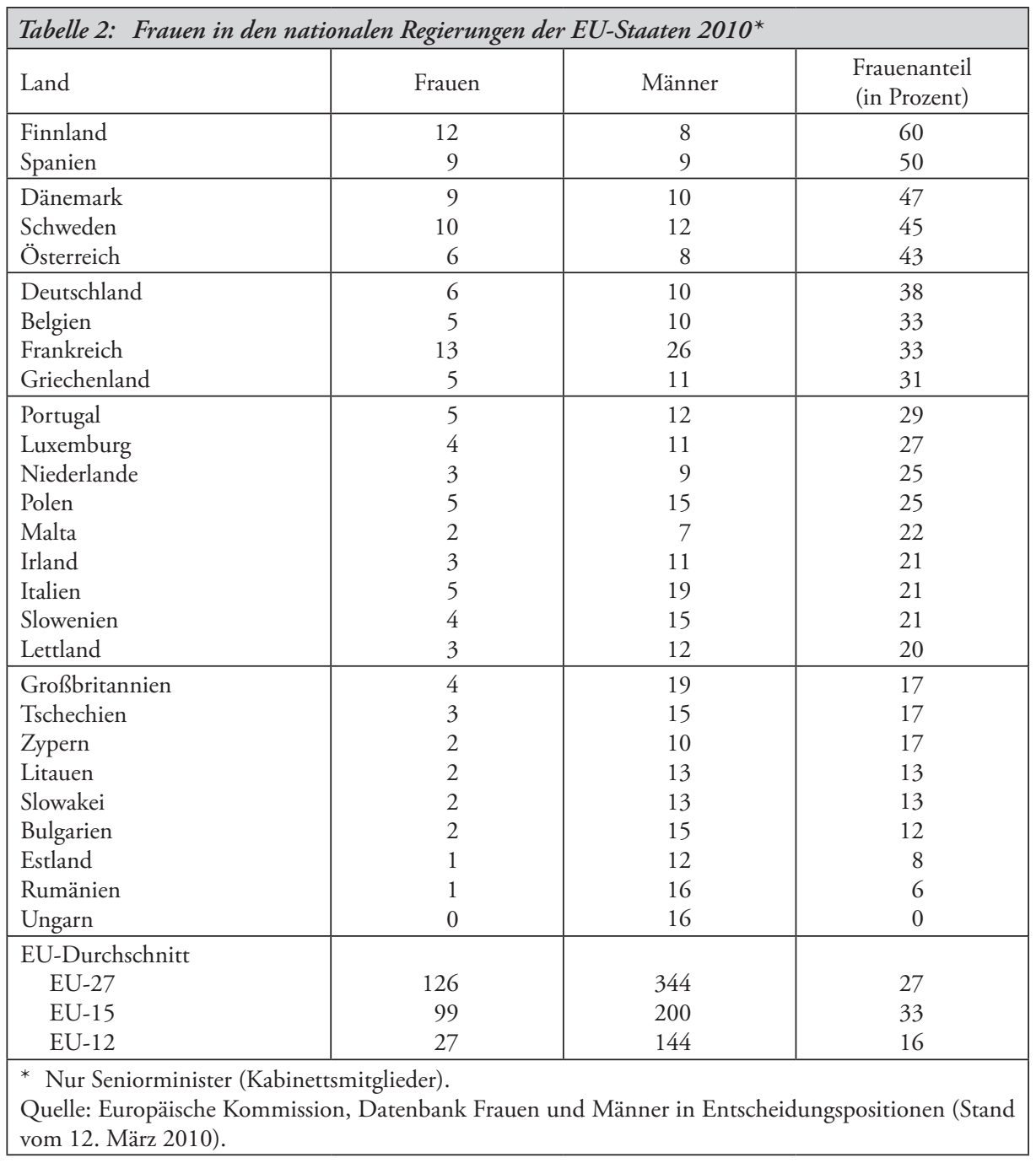

Vertretung. Typisch ist aber auch hier eine enorme Spannbreite der Frauenanteile, die von 60 Prozent (Finnland) bis 0 Prozent (Ungarn) reicht (vgl. Tabelle 2).

Während Frauen allein in Finnland das Kabinett dominieren, kommen sie in Spanien immerhin auf eine paritätische Vertretung (50 Prozent). Deutlich über 40 Prozent erreichen Frauen aber auch in den Regierungen von Dänemark (47), Schweden (45) sowie Österreich (43), und gut beziehungsweise knapp ein Drittel Ministerinnen finden sich in Deutschland (38), Belgien (33), Frankreich (33) und Griechenland (31). Über dem EUDurchschnitt liegt gleichfalls noch Portugal (29), während Luxemburg (27) dem durchschnittlichen Wert exakt entspricht. Alle weiteren 16 Staaten bleiben bei der Vertretung von Frauen in den Regierungen unter dem europaweiten Durchschnitt, wobei - wie schon beim parlamentarischen Frauenanteil - hierzu alle Beitrittsstaaten zählen. Hier beträgt die weibliche Regierungsbeteiligung im Durchschnitt nur rund 16 Prozent, in den alten EU- 
Staaten dagegen 33 Prozent. Der so genannte gender gap zwischen alten und neuen EUStaaten fällt somit bei der Regierungsbeteiligung erkennbar größer aus als bei der parlamentarischen Vertretung (17 beziehungsweise 11 Prozentpunkte).

Der Anteil der Ministerinnen liegt in 14 der 27 EU-Staaten über dem der Parlamentarierinnen. Besonders markante Differenzen ergeben sich dabei für Finnland (+ 20 Prozentpunkte), Österreich $(+15)$, Frankreich $(+14)$, Griechenland $(+14)$, Spanien $(+13)$ und Malta (+ 13 Prozentpunkte). Erheblich schlechter repräsentiert als im Parlament sind Frauen dagegen in der Regierung der Niederlande (-17) sowie in den Kabinetten von Estland (-15) und Bulgarien (- 9 Prozentpunkte). Eine Erklärung für diese Differenzen dürfte in der parteipolitischen Zusammensetzung der Regierungen zu sehen sein: So sind Mitterechts Parteien beziehungsweise Koalitionen zumeist weniger frauenfreundlich als ideologisch eher links stehende Parteien. ${ }^{13}$ Generell ist jedoch festzuhalten, dass ein hoher Frauenanteil unter den Abgeordneten in der Regel auch einen hohen Frauenanteil in der Regierung nach sich zieht. Von den aktuell sieben EU-Staaten mit einem parlamentarischen Frauenanteil von mindestens 30 Prozent verfügen sechs auch bei den Kabinettsmitgliedern über einen solchen Anteil. ${ }^{14}$

Über die Ressorts der Ministerinnen gibt die eingeführte BEIS-Typologie ${ }^{15}$ Auskunft: So waren 2007 rund 37 Prozent für Soziokulturelle Aufgaben zuständig, gefolgt von Infrastruktur und Basisaufgaben (je 21 Prozent) sowie Wirtschaft (18 Prozent). Damit ist das traditionelle Muster, wonach Frauen eher die als „weich“ geltenden Ministerien übertragen werden, inzwischen zwar abgeschwächt, europaweit aber noch immer erkennbar. ${ }^{16}$

Im Kreis der Regierungschefs schließlich sind Männer nach wie vor nahezu unter sich; lediglich in Deutschland, Finnland und der Slowakei führen Frauen derzeit eine Regierung an (11 Prozent).

\subsection{Die Repräsentation von Frauen in den europäischen Organen/Institutionen}

Auf europäischer Ebene wird allein das Europäische Parlament (EP) direkt gewählt und verfügt damit über eine starke Legitimation. Auch wenn innerhalb der Politikwissenschaft keine Einigkeit darüber besteht, ob das EP „als ,normale Legislative’ mit einigen Besonderheiten oder als Institution ,sui generis' einzuordnen ist" 17 , hat es sich im europäischen Mehrebenensystem inzwischen zu einem eigenständigen Organ politischer Gestaltung entwickelt. Von Anfang an fiel es zudem durch eine vergleichsweise hohe Repräsentation von Frauen auf; so betrug der Anteil der weiblichen Abgeordneten im ersten direkt gewählten EP 1979 bereits rund 17 Prozent und stieg bis 2004 nahezu kontinuierlich auf 30 Prozent

13 Für einen eindrucksvollen Beleg vgl. Drude Dahlerup / Lenita Freidenvall, Geschlechterquoten bei Wahlsystemen und ihre Umsetzung in Europa, Brüssel 2008, S. 24 ff.

14 Vgl. hierzu auch Europäische Kommission, Frauen in der europäischen Politik, a.a.O. (Fn. 6), S. 47.

15 Die vier Kategorien der BEIS-Typologie sind: Basisaufgaben: Äußeres und Inneres, Verteidigung, Justiz; Wirtschaft: Finanzen, Handel, Industrie, Landwirtschaft; Infrastruktur: Verkehr, Kommunikation, Umwelt; Soziokulturelle Aufgaben: Soziales, Beschäftigung, Gesundheit, Kinder, Familie, Jugend, Senioren, Bildung, Wissenschaft, Kultur, Arbeit, Sport usw. Vgl. Europäische Kommission, Frauen und Männer in Entscheidungspositionen 2007, Luxemburg 2008, S. 26 f.

16 Vgl. ebenda, S. $27 \mathrm{ff}$.

17 Wolfgang Wessels, Das politische System der Europäischen Union, Wiesbaden 2008, S. 119. 


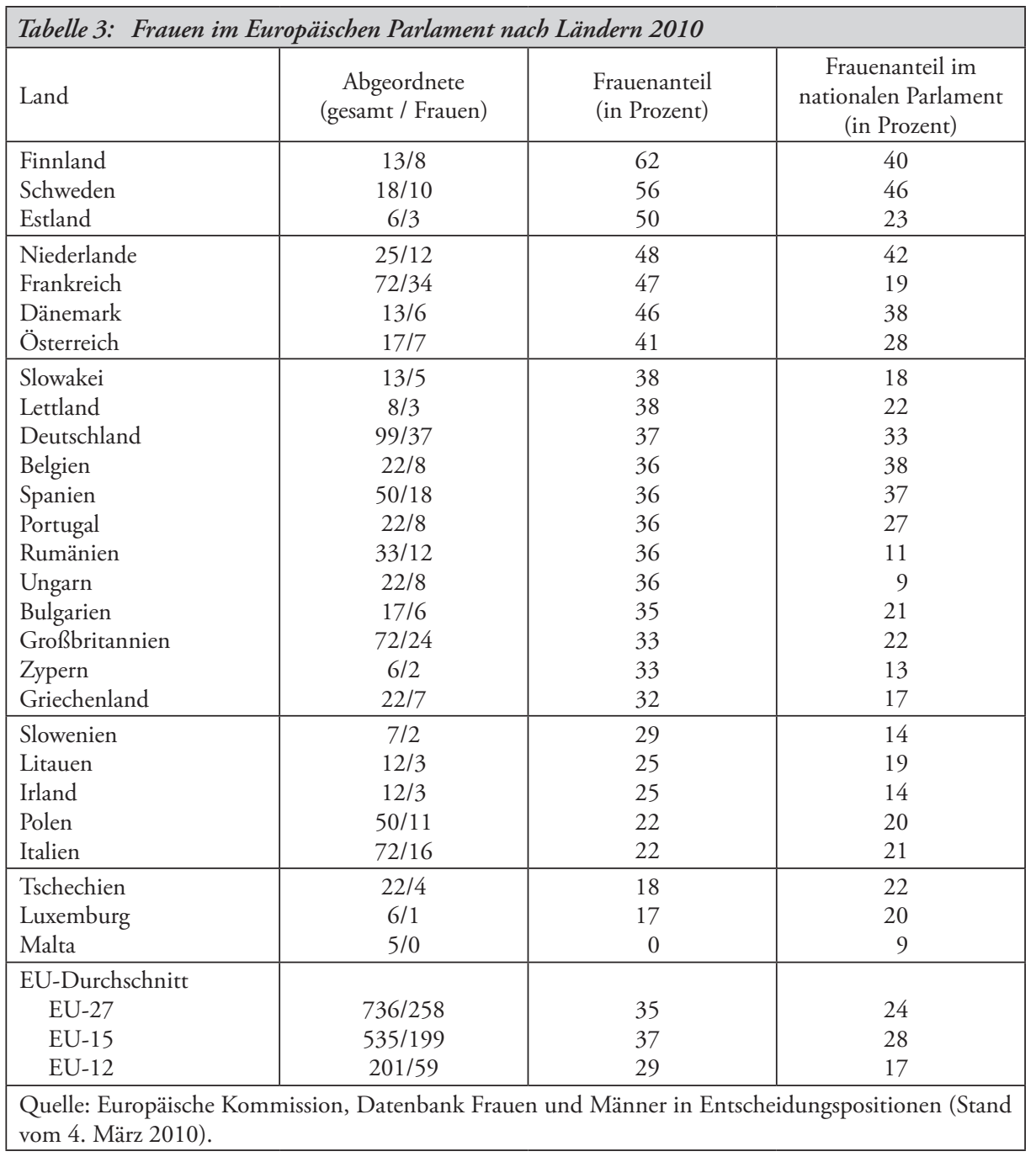

an. Die siebte Wahl vom Juni 2009 brachte eine weitere Steigerung auf 35 Prozent. Mit nur wenigen Ausnahmen (Belgien, Spanien, Luxemburg und Malta) sind Frauen damit im EP erkennbar besser repräsentiert als im jeweiligen nationalen Parlament (vgl. Tabelle 3).

Im Ländervergleich zeigen sich allerdings auch auf dieser Ebene teilweise erhebliche Unterschiede. Auf besonders hohe Frauenanteile können wiederum die nordischen Staaten Finnland (62 Prozent) und Schweden (56) verweisen, aber auch das kleine Estland kommt auf 50 Prozent. Weitere zwölf Staaten liegen mit ihren Frauenanteilen im Europäischen Parlament über dem Durchschnitt, während Bulgarien genau 35 Prozent erreicht. Unterdurchschnittliche Frauenanteile finden sich demgegenüber in elf Staaten, wobei Tschechien (18), Luxemburg (17) und Malta (0 Prozent) die Schlusslichter bilden.

Unterscheidet man wiederum zwischen alten und neuen EU-Mitgliedern, dann zeigt sich ein interessantes Phänomen: Während innerhalb der EU-15 im Durchschnitt ein Frau- 
enanteil von 37 Prozent im EP erreicht wird, beträgt er im Kreis der EU-12 29 Prozent. Mit acht Prozentpunkten fällt damit die Differenz zwischen alten und neuen EU-Staaten erheblich geringer aus als auf der nationalen Ebene. Aus Sicht der Beitrittsländer bedeutet dieses zugleich, dass Frauen im EP mit Abstand stärker repräsentiert sind als in den nationalen Parlamenten und Regierungen (vgl. Tabelle 4). Diese Feststellung gilt auch für die vorherige Wahl zum EP, an der die neuen Staaten erstmals teilnahmen. Nach Stockemer liegt genau darin auch die Erklärung: „The fact that these new EU members voted for the first time in European parliamentary elections in 2004 (...) constituted a good opportunity structure for women. Women did not have to face the problem of incumbency. Party elites could respond favourably to women's demands for adequate inclusion without requiring male party dignitaries to step aside." 18

\begin{tabular}{|c|c|c|c|c|}
\hline \multicolumn{5}{|c|}{$\begin{array}{l}\text { Tabelle 4: Frauenanteile auf nationaler und europäischer Ebene } 2010 \text { (EU-Durchschnitt, } \\
\text { in Prozent) }\end{array}$} \\
\hline EU-Durchschnitt & $\begin{array}{l}\text { Nationale } \\
\text { Parlamente }\end{array}$ & $\begin{array}{c}\text { Nationale } \\
\text { Regierungen }\end{array}$ & $\begin{array}{c}\text { Europäisches } \\
\text { Parlament }\end{array}$ & $\begin{array}{l}\text { Europäische } \\
\text { Kommission }\end{array}$ \\
\hline EU-27 & 24 & 27 & 35 & 33 \\
\hline EU-15 & 28 & 33 & 37 & 47 \\
\hline EU-12 & 17 & 16 & 29 & 17 \\
\hline $\begin{array}{l}\text { Differenz EU-15/ } \\
\text { EU-12 (Prozentpunkte) }\end{array}$ & 11 & 17 & 8 & 30 \\
\hline
\end{tabular}

Fasst man die weiteren Institutionen auf europäischer Ebene ins Auge, stellen Frauen in der als besonders machtvoll geltenden Europäischen Kommission gegenwärtig ein Drittel der Mitglieder (2005: 28 Prozent). Unter den 27 Kommissaren finden sich für die alten EUStaaten sieben, für die neuen zwei Frauen. In den beratenden Ausschüssen, nämlich dem Ausschuss der Regionen sowie dem Wirtschafts- und Sozialausschuss, sind Frauen derzeit nur zu 19 beziehungsweise 23 Prozent vertreten.

Als eher deprimierendes Fazit der Bestandsaufnahme bleibt festzuhalten: Auch wenn sich in den letzten Jahren die politische Repräsentation von Frauen auf nationaler wie europäischer Ebene im Durchschnitt leicht erhöht hat, liegt Geschlechterparität europaweit noch in weiter Ferne. Der im Ländervergleich allerdings sehr unterschiedliche Stand der politischen Gleichstellung wirft nun die Frage auf, welche Erklärungen es dafür aus komparativer Sicht gibt. Aufgrund des Forschungsstands steht die parlamentarische Repräsentation dabei im Mittelpunkt.

\section{Erklärungsansätze der parlamentarischen Repräsentation von Frauen in den EU-Staaten}

In der wissenschaftlichen Literatur besteht weitgehend Konsens darüber, dass institutionelle, sozialstrukturelle und kulturelle Faktoren die politische Repräsentation von Frauen be-

18 Daniel Stockemer, Women's Representation in Europe - A Comparison Between the National Parliaments and the European Parliament, in: Comparative European Politics, 6. Jg. (2008), H. 6, S. $463-485$, S. 479. 
einflussen. Divergierende Auffassungen gibt es jedoch bei der Bedeutung, die den jeweiligen Faktoren im - oftmals weltweiten - Ländervergleich zugemessen wird. Während einige Forscher vor allem institutionelle Rahmenbedingungen als entscheidend für die parlamentarische Repräsentation von Frauen ansehen ${ }^{19}$, schreiben andere wiederum den kulturellen Geschlechterrollenbildern eine herausragende Bedeutung zu. ${ }^{20}$ Gleichwohl konstituieren die von Beate Hoecker und Gesine Fuchs (2004) im „magischen Dreieck“ zusammengefassten Erklärungsvariablen auch für den EU-Vergleich den adäquaten Analyserahmen, und es ist somit zu fragen, welche Gemeinsamkeiten beziehungsweise Unterschiede zwischen den europäischen Staaten im Hinblick auf die drei Faktoren bestehen. ${ }^{21}$

\subsection{Institutionelle Faktoren}

$\mathrm{Zu}$ den institutionellen Bedingungen zählen neben dem Parteiensystem insbesondere die politischen Rekrutierungsmuster sowie das Wahlsystem. Wie zahlreiche Studien belegen, begünstigen Verhältniswahlsysteme die Kandidaturen von Frauen, während Mehrheitswahlsysteme diese eher erschweren. ${ }^{22}$ Die aktuelle Rangordnung bestätigt dieses Ergebnis; die Staaten mit einer hohen parlamentarischen Repräsentation von Frauen wählen ihre Volksvertretungen fast durchgehend nach dem Verhältniswahlrecht (Ausnahme: Spanien). Großbritannien und Frankreich dagegen, wo die Mehrheitswahl angewandt wird, liegen mit ihren Frauenanteilen im House of Commons beziehungsweise der Assemblée Nationale nur im mittleren/unteren Bereich. Demgegenüber verbinden so genannte Mischwahlsysteme Elemente der Verhältnis- und der Mehrheitswahl und können näher an der Verhältniswahl (zum Beispiel Portugal) oder an der Mehrheitswahl (zum Beispiel Irland) liegen. ${ }^{23}$ Für die Kandidaturen von Frauen erweisen sich diese Mischwahlsysteme gleichfalls als eher ungünstig (vgl. Tabelle 5).

Der Grund für die geringeren Nominierungschancen von Frauen im Falle eines Mehrheitswahlrechts wird allgemein in der starken Personenorientierung gesehen. Im Unterschied zur Listenwahl muss sich die Wählerschaft für einen Kandidaten beziehungsweise eine Kandidatin im Wahlkreis direkt entscheiden. Nach wie vor bestehende Zweifel an der politischen Kompetenz von Frauen, aber auch der harte innerparteiliche Konkurrenzkampf um sichere Wahlkreise mindern die Chancen von Frauen, überhaupt aufgestellt zu werden.

19 So beispielsweise Ian McAllister / Donley T. Studlar, Electoral Systems and Women's Representation. A Long-Term Perspective, in: Representation, 39. Jg. (2002), H. 1, S. 3 - 13; ebenso Aili Mari Tripp / Alice Kang, The Global Impact of Quotas. On the Fast Track to Increased Female Legislative Representation, in: Comparative Political Studies, 41. Jg. (2008), H. 3, S. 338 - 361.

20 Vgl. hierzu Ronald Inglehart / Pippa Norris, Rising Tide. Gender Equality And Cultural Change Around the World, Cambridge 2003; siehe auch Beate Hoecker, a.a.O. (Fn. 4). Die kontroversen Forschungsergebnisse dürften sich zumindest teilweise durch unterschiedliche methodische Ansätze beziehungsweise Operationalisierungen sowie Differenzen hinsichtlich der geographisch einbezogenen Länder erklären.

21 Ausführlich zu den Einflussfaktoren vgl. Beate Hoecker / Gesine Fuchs, a.a.O. (Fn. 4), S. 13 ff.

22 Vgl. Wilma Rule / Joseph F. Zimmerman (Hrsg.), Electoral Systems. Their Impact on Minorities and Women, Westport 1994; Ian McAllister / Donley T. Studlar, a.a.O. (Fn. 19).

23 Vgl. hierzu Eckhard Jesse, Wahlsysteme und Wahlrecht, in: Oscar W. Gabriel / Sabine Kropp (Hrsg.), a.a.O. (Fn. 7), S. 297 - 322, S. 308. 
Gleichwohl lässt sich auch ein Mehrheitswahlsystem frauenfreundlicher gestalten, beispielsweise durch die Vergabe von sicheren Wahlkreisen an Frauen. Voraussetzung ist allerdings der Wille, Frauen gleichberechtigt am politischen Prozess zu beteiligen. Fehlt dieser Wille, ist auch ein Verhältniswahlsystem kein Garant für eine hohe Präsenz von Frauen im Parlament. Zwar bieten Verhältniswahlsysteme den Vorzug einer Quotierung der Parteilisten zu Gunsten von Frauen, allerdings müssen diese Quoten einen relevanten Anteil fixieren, verbindlich sein und sich zudem explizit auf die aussichtsreichen Listenplätze beziehen. Diese Bedingungen aber werden von den Parteien in Europa längst nicht immer erfüllt; insofern führt eine solche positive Diskriminierung auch nicht automatisch zu einem hohen Frauenanteil im Parlament. ${ }^{24}$ Umgekehrt muss der Verzicht auf eine Quotenregelung nicht gleichbedeutend sein mit einer nur marginalen Vertretung von Frauen, wie die Beispiele Finnland und Dänemark zeigen (vgl. Tabelle 5).

Als Alternative zu den freiwilligen parteiinternen Quoten bieten sich gesetzliche Wahlquoten an. Fünf EU-Staaten (Frankreich, Belgien, Spanien, Portugal und Slowenien) haben inzwischen zu diesem Instrument gegriffen, wobei der Erfolg unterschiedlich ist, wie die folgenden drei Beispiele zeigen. ${ }^{25}$

(1) Nach dem belgischen Wahlgesetz von 2002 darf sich die Zahl der Kandidaten jedes Geschlechts auf den Listen um nicht mehr als eins unterscheiden. Die Personen auf dem ersten und zweiten Listenplatz müssen zudem unterschiedlichen Geschlechts sein. Diese gesetzliche Bestimmung führte zu einem sprunghaften Anstieg des Frauenanteils im belgischen Abgeordnetenhaus, dennoch blieb dieser deutlich unter 50 Prozent. Der Grund für den nur teilweisen Erfolg liegt darin, dass eine alternierende Aufstellung ab dem dritten Platz im Gesetz nicht vorgeschrieben ist, was den Parteien weiterhin die Möglichkeit gibt, ihre Kandidatinnen eher am Ende der Liste zu platzieren. ${ }^{26}$

(2) In Spanien sieht das Wahlgesetz von 2007 vor, dass jedes Geschlecht mit mindestens 40 und höchstens 60 Prozent auf den Wahllisten vertreten sein darf. Auf nationaler Ebene kam es allerdings zu keiner weiteren Erhöhung des parlamentarischen Frauenanteils, da dieser bereits zuvor - aufgrund von parteiinternen Quoten - vergleichsweise hoch ausfiel. ${ }^{27}$

(3) In Frankreich legt das „Paritätsgesetz“ aus dem Jahr 2000 fest, dass bei Listenwahlen nach dem Verhältniswahlrecht (unter anderem Kommunalwahlen und Wahlen zum EP) jedes Geschlecht mit 50 Prozent vertreten sein muss, wobei - seit den Reformen von 2003 und 2007 - eine strikt alternierende Reihenfolge der Kandidierenden gefordert ist. Wird diese Vorgabe nicht eingehalten, ist die Liste ungültig. Für Wahlen nach dem Mehrheitswahlrecht (unter anderem Nationalwahlen) müssen die Parteien gleichfalls je 50 Prozent männliche und weibliche Kandidaten aufstellen, andernfalls werden sie mit einem Bußgeld belegt. Diese unterschiedlichen Sanktionen hatten zur Folge, dass auf kommunaler Ebene, aber auch im Europäischen Parlament der Frauenanteil eindrucksvoll in die Höhe kletterte, während er in der Assemblée Nationale enttäuschend gering blieb. Nach Mariette Sineau

24 Vgl. Drude Dahlerup / Lenita Freidenvall, a.a.O. (Fn. 13), S. 27 ff.

25 In Portugal erhöhte sich mit Einführung der gesetzlichen Wahlquote der Frauenanteil im Parlament deutlich, in Slowenien dagegen blieb er nahezu unverändert niedrig. Vgl. zur Ausgestaltung der Wahlquoten ebenda, S. $39 \mathrm{ff}$.

26 Vgl. Petra Meier, Belgien: eine bewährte Vorgehensweise ins rechte Licht gerückt, in: Drude Dahlerup / Lenita Freidenvall (Hrsg.), a.a.O. (Fn. 13), S. 40 - 44.

27 Vgl. Christina Alnevall, Spanien - auf dem Weg zu einer besser gleichgestellten Gesellschaft, in: Drude Dablerup / Lenita Freidenvall (Hrsg.), a.a.O. (Fn. 13), S. 101 - 108. 
Tabelle 5: Einflussfaktoren der parlamentarischen Repräsentation von Frauen in den EU-Mitgliedstaaten

\begin{tabular}{|c|c|c|c|c|c|c|c|c|}
\hline \multirow[b]{2}{*}{ Land } & \multicolumn{3}{|c|}{$\begin{array}{l}\text { Parlamentarische } \\
\text { Repräsentation }^{1}\end{array}$} & \multicolumn{3}{|c|}{$\begin{array}{c}\text { Einführung } \\
\text { Frauenwahlrecht }{ }^{2}\end{array}$} & \multicolumn{2}{|c|}{$\begin{array}{l}\text { Zustimmung zur traditio- } \\
\text { nellen Arbeitsteilung }\end{array}$} \\
\hline & hoch & mittel & niedrig & früh & mittel & spät & $\begin{array}{l}\text { mehrheitlich } \\
\text { Zustimmung }\end{array}$ & $\begin{array}{c}\text { mehrheitlich } \\
\text { Ablehnung }\end{array}$ \\
\hline Schweden & $\bullet$ & & & & $\cdot \mathrm{z}$ & & & • \\
\hline Niederlande & $\bullet$ & & & & • & & & $\bullet$ \\
\hline Finnland & - & & & $\cdot \mathrm{z}$ & & & • & \\
\hline Dänemark & - & & & & $\cdot \mathrm{z}$ & & & - \\
\hline Belgien & $\bullet$ & & & & & • & & - \\
\hline Spanien & $\bullet$ & & & & $\bullet$ & & & - \\
\hline Deutschland & • & & & & • & & - (West) & - (Ost) \\
\hline Österreich & & - & & & - & & - & \\
\hline Portugal & & • & & & & • & • & \\
\hline Estland & & $\bullet$ & & & $\bullet$ & & $\bullet$ & \\
\hline Großbritannien & & - & & & • & & & • \\
\hline Lettland & & • & & & - & & • & \\
\hline Tschechien & & - & & & • & & • & \\
\hline Bulgarien & & • & & & & • & • & \\
\hline Italien & & • & & & & - & • & \\
\hline Luxemburg & & $\bullet$ & & & • & & & • \\
\hline Polen & & • & & & • & & • & \\
\hline Litauen & & & • & & $\bullet$ & & $\bullet$ & \\
\hline Frankreich & & & • & & & • & & $\bullet$ \\
\hline Slowakei & & & $\bullet$ & & • & & • & \\
\hline Griechenland & & & • & & & - & - & \\
\hline Irland & & & $\bullet$ & & $\bullet$ & & $\bullet$ & \\
\hline Slowenien & & & • & & & • & - & \\
\hline Zypern & & & - & & & $\bullet$ & • & \\
\hline Rumänien & & & • & & & • & • & \\
\hline Ungarn & & & • & & & - & - & \\
\hline Malta & & & • & & & - & - & \\
\hline \multicolumn{9}{|c|}{$\begin{array}{l}\text { Die parlamentarische Repräsentation bezieht sich auf das Nationalparlament (zum jeweiligen Stand vgl. } \\
\text { Tabelle 1). Einteilung: ,hoch“ = über } 30 \text { Prozent; „mittel“= } 20 \text { bis } 30 \text { Prozent; „niedrig“ = unter } 20 \text { Prozent. } \\
2 \text { Einführung des Frauenwahlrechts: „früh“ = vor dem 1. Weltkrieg; „mittel“ = nach dem } 1 \text {. Weltkrieg; „,spät“ } \\
\text { = nach } 1940 ; \mathrm{z}=\text { zeitgleiche Einführung mit dem Männerwahlrecht. } \\
3 \text { Indikator: „Idealerweise sollte die Frau zu Hause bleiben und sich um die Kinder kümmern, während der } \\
\text { Mann arbeitet.“ }\end{array}$} \\
\hline
\end{tabular}




\begin{tabular}{|c|c|c|c|c|c|c|c|c|}
\hline \multicolumn{9}{|c|}{ Fortsetzung Tabelle 5} \\
\hline & \multicolumn{3}{|c|}{ Wahlsystem } & \multicolumn{2}{|c|}{$\begin{array}{c}\text { Geschlechterquoten für } \\
\text { öffentliche Kandidaturen }\end{array}$} & \multicolumn{3}{|c|}{$\begin{array}{l}\text { Beschäftigungsquote } \\
\text { von Frauen }^{4}\end{array}$} \\
\hline Land & $\begin{array}{l}\text { Verhältnis- } \\
\text { wahlsystem }\end{array}$ & $\begin{array}{l}\text { Mehrheits- } \\
\text { wahlsystem }\end{array}$ & $\begin{array}{c}\text { Mischwahl- } \\
\text { system }\end{array}$ & $\begin{array}{l}\text { ja (gesetzlich/ } \\
\text { parteiintern) }\end{array}$ & nein & hoch & mittel & niedrig \\
\hline Schweden & • & & & $\cdot p$ & & $\bullet$ & & \\
\hline Niederlande & - & & & $\cdot p$ & & $\bullet$ & & \\
\hline Finnland & • & & & & $\bullet$ & $\bullet$ & & \\
\hline Dänemark & $\bullet$ & & & & • & $\bullet$ & & \\
\hline Belgien & - & & & $\cdot \mathrm{g}$ & & & $\bullet$ & \\
\hline Spanien & & & $\bullet$ & - $\mathrm{g}$ & & & $\bullet$ & \\
\hline Deutschland & • & & & $\cdot p$ & & $\bullet$ & & \\
\hline Österreich & $\bullet$ & & & $\cdot \mathrm{p}$ & & $\bullet$ & & \\
\hline Portugal & & & $\bullet$ & $\cdot g$ & & $\bullet$ & & \\
\hline Estland & $\bullet$ & & & & $\bullet$ & $\bullet$ & & \\
\hline Großbritannien & & $\bullet$ & & $\cdot p$ & & $\bullet$ & & \\
\hline Lettland & - & & & & • & & - & \\
\hline Tschechien & $\bullet$ & & & $\cdot \mathrm{p}$ & & & • & \\
\hline Bulgarien & $\bullet$ & & & & $\bullet$ & & $\bullet$ & \\
\hline Italien & & & $\bullet$ & $\cdot \mathrm{p}$ & & & & $\bullet$ \\
\hline Luxemburg & & & - & $\cdot \mathrm{p}$ & & & - & \\
\hline Polen & - & & & $\cdot \mathrm{p}$ & & & - & \\
\hline Litauen & & & $\bullet$ & $\cdot \mathrm{p}$ & & $\bullet$ & & \\
\hline Frankreich & & $\bullet$ & & $\cdot g$ & & $\bullet$ & & \\
\hline Slowakei & • & & & & $\bullet$ & & - & \\
\hline Griechenland & & & $\bullet$ & $\cdot \mathrm{p}$ & & & & $\bullet$ \\
\hline Irland & & & $\bullet$ & & $\bullet$ & & $\bullet$ & \\
\hline Slowenien & $\bullet$ & & & $\cdot g$ & & $\bullet$ & & \\
\hline Zypern & & & $\bullet$ & & $\bullet$ & • & & \\
\hline Rumänien & & & - & $\cdot \mathrm{p}$ & & & - & \\
\hline Ungarn & & & $\bullet$ & $\cdot \mathrm{p}$ & & & & $\bullet$ \\
\hline Malta & & & $\bullet$ & $\cdot \mathrm{p}$ & & & & $\bullet$ \\
\hline \multicolumn{9}{|c|}{$\begin{array}{l}4 \text { Beschäftigungsquote der Frauen zwischen } 15 \text { und } 64 \text { Jahren (Stand: 2009); Einteilung: „hoch“ = über } 60 \text { Pro- } \\
\text { zent; „mittel“ }=50 \text { bis } 60 \text { Prozent "niedrig“ = } 40 \text { bis } 50 \text { Prozent. } \\
\text { Quellen: Eigene Zusammenstellung. Einführung Frauenwahlrecht und Geschlechterquoten nach: Europäische } \\
\text { Kommission, Frauen in der europäischen Politik, Luxemburg 2009, S. 20, S. 32; Indikator: „Zustimmung zur } \\
\text { traditionellen Arbeitsteilung“ nach Eurobarometer } 65.1 \text { (Frühjahr 2006); Wahlsystem nach Eckhard Jesse, Wahl- } \\
\text { systeme und Wahlrecht, in: Oscar W. Gabriel / Sabine Kropp (Hrsg.), Die EU-Staaten im Vergleich, Wiesbaden } \\
\text { 2008, S. 299 - 322, S. 307; Beschäftigungsquote nach Eurostat, http://epp.eurostat.ec.europa.eu/tgm/table.do?t } \\
\text { ab=table\&plugin=1\&language=de\&pcode=tsiem010 (Abruf am 24. Juli 2010). }\end{array}$} \\
\hline
\end{tabular}


„ziehen es die Parteien - insbesondere die des rechten Spektrums - vor, Bußgelder zu zahlen, statt sich auf die Nominierung guter weiblicher Neulinge zu einigen" ${ }^{28}$.

Wie die Beispiele belegen, spielen neben der Fixierung eines relevanten Geschlechteranteils auch Rangfolgebestimmungen (bei Listenwahlen) sowie spürbare Sanktionen eine entscheidende Rolle für den Erfolg gesetzlicher Wahlquoten. Dass dieser Erfolg aber längst nicht immer intendiert ist, verweist auf die Bedeutung kultureller Einstellungsmuster.

\subsection{Kulturelle Faktoren}

Nach der klassischen Definition von Gabriel Almond und Sidney Verba ${ }^{29}$ umfasst die Politische Kultur als subjektive Dimension die Gesamtheit aller politisch relevanten Meinungen, Einstellungen und Werte der Mitglieder einer Nation. Gesellschaftliche Orientierungen gegenüber der politischen Rolle von Frauen sind somit Bestandteil der Politischen Kultur und variieren je nach Kulturgefüge.

Die Annahme, dass für Länder mit eher traditionellen Geschlechterrollenbildern auch eine nur geringe parlamentarische Vertretung typisch ist, während dagegen Staaten mit einer eher egalitären Kultur eine vergleichsweise hohe Repräsentation von Frauen aufweisen, kann als weitgehend bestätigt gelten. ${ }^{30} \mathrm{Wie}$ vergleichende Studien belegen, lassen sich insbesondere die nordischen Staaten (vor allem Schweden, Dänemark und das Nicht-EULand Norwegen) durch eine ausgesprochen geschlechteregalitäre Ausrichtung kennzeichnen, die mit einer staatlich geförderten, hohen und kontinuierlichen Erwerbsbeteiligung von Frauen einhergeht. ${ }^{31}$ Allein diese Staaten haben zudem Männern und Frauen zeitgleich die staatsbürgerlichen Rechte gegeben (Ausnahme: Norwegen); die politische Sphäre konnte sich hier somit von Anfang an nicht als eine rein männliche Domäne ausbilden. Eine überraschende - Ausnahme stellt allerdings Finnland dar, das einer neueren Untersuchung von Detlev Lück zufolge anscheinend nicht in vergleichbarer Weise wie die anderen nordeuropäischen Länder über eine Kultur der Geschlechtergleichheit verfügt. ${ }^{32}$

28 Mariette Sineau, Frankreich: gesetzlich geregelte Gleichstellung - das „Parité--Gesetz, in: Drude Dablerup / Lenita Freidenvall (Hrsg.), a.a.O. (Fn. 13), S. 55 - 65, S. 63. Vgl. auch Cécile Teissier, Gesetzliche Verwirklichung der Geschlechterparität bei französischen Wahlmandaten - Erste Anwendungserfahrungen, in: ZParl, 33. Jg. (2002), H. 1, S. 115 - 124.

29 Vgl. Gabriel Almond / Sidney Verba, The Civic Culture. Political Attitudes and Democracy in 5 Nations, Princeton 1963.

30 Eine Ausnahme stellen die Ergebnisse von Stockemer dar, wonach die Politische Kultur erstaunlicherweise keinen Einfluss auf die parlamentarische Repräsentation von Frauen in den 27 EUStaaten ausübt. Entscheidend sind seiner Regressionsanalyse zufolge vielmehr die zeitliche Dauer des Frauenwahlrechts, das Wahlsystem sowie der Frauenanteil in beruflichen Führungspositionen. Ganz wohl ist dem Autor aber selbst nicht bei diesem Resultat, denn selbstkritisch verweist er auf die „sub-optimal operationalization“ der Politischen Kultur. Vgl. Daniel Stockemer, Why are there Differences in the Political Representation of Women in the 27 Countries of the European Union?, in: Perspectives on European Politics and Society, 8. Jg. (2007), H. 4, S. 476 - 493.

31 Vgl. Christina Bergqvist u.a. (Hrsg.), Equal Democracies? Gender and Politics in the Nordic Countries, Oslo 1999; Lenita Freidenvall / Drude Dahlerup / Hege Skjeie, The Nordic Countries: An Incremental Model, in: Drude Dahlerup (Hrsg.), Women, Quotas and Politics, New York 2006, S. $55-82$.

32 Vgl. Detlev Lück, Der zögernde Abschied vom Patriarchat. Der Wandel von Geschlechterrollen im internationalen Vergleich, Berlin 2009, S. 264 f. 
Egalitäre Einstellungen bestehen nach Lück darüber hinaus aber auch seit längerem in Großbritannien und den Niederlanden. Für die süd- und mitteleuropäischen Staaten konstatiert der Autor im Zeitverlauf (1988 bis 2002) gleichfalls einen Wandel hin zu mehr Egalität. Danach legen diese post-industriellen Gesellschaften „ihre Vorstellungen ab, dass es unterschiedliche Zuständigkeiten geben müsse, dass die Sphäre der Erwerbsarbeit grundsätzlich dem Mann und die Hausarbeit und Kindererziehung der Frau vorbehalten bleiben müsse. Stattdessen gewinnen Leitbilder an Kraft, die eine gemeinsame und gleichberechtigte Zuständigkeit beider Sphären definieren "33. In der politischen Sphäre hat sich dieser Einstellungswandel allerdings noch nicht durchgreifend niedergeschlagen.

Während die westlichen Gesellschaften somit bereits egalitäre Einstellungen vertreten beziehungsweise sich auf dem Weg dorthin befinden, dominieren in den postkommunistischen, osteuropäischen Ländern noch traditionelle Geschlechterrollenbilder. Trotz Integration in die Erwerbsarbeit sind Frauen hier allein zuständig für Hausarbeit und Kindererziehung, was eine enorme Doppelbelastung bedeutet und die Chancen für politisches Engagement allein zeitlich stark mindert. ${ }^{34}$ Allerdings wird Politik als eine männliche Domäne überwiegend akzeptiert, und im EU-Vergleich fällt die osteuropäische Unterstützung für eine dringende Erhöhung des Frauenanteils in den nationalen Parlamenten folglich nur gering aus. ${ }^{35}$ Nach Hoecker / Fuchs steht fest, „dass in der patriarchalen politischen Kultur mit der wichtigste Grund für die nur geringe politische Partizipation wie Repräsentation von Frauen in den Transformationsstaaten liegt" ${ }^{\text {"36. }}$.

Die generellen empirischen Befunde zu den Geschlechterrollenbildern in Europa spiegeln sich in den Zuordnungen in Tabelle 5 allerdings nur zum Teil wider („Zustimmung zur traditionellen Arbeitsteilung"). Weitgehende Übereinstimmung mit den Befunden von Lück besteht für die Spitzengruppe sowie die osteuropäischen Länder. Während erstere das traditionelle Rollenmodell fast durchgängig ablehnen (Ausnahmen: Finnland und Westdeutschland), stimmen alle Transformationsstaaten dem „Ernährermodell“ mehrheitlich zu. Der von Lück konstatierte Einstellungswandel innerhalb der etablierten westlichen Demokratien dagegen zeigt sich - neben Belgien und Spanien - nur noch für Großbritannien, Frankreich und Luxemburg; die südeuropäischen Staaten Italien, Portugal und Griechenland sowie Österreich und Irland befürworten demgegenüber die traditionelle Arbeitsteilung zwischen den Geschlechtern. Diese Abweichungen können methodisch begründet sein; möglicherweise aber deuten sie auch auf eine Re-Traditionalisierung der Geschlechterrollenbilder hin. Erst weitere Forschungen werden hier zu gesicherten Ergebnissen führen.

33 Ebenda, S. 277 f.

34 Vgl. ebenda, S. 269 f.; siehe auch Beate Hoecker / Gesine Fuchs, a.a.O. (Fn. 9), S. 290 ff. sowie die Länderstudien in Marilyn Rueschemeyer / Sharon L. Wolchik (Hrsg.), a.a.O. (Fn. 4).

35 Vgl. Special Eurobarometer 326, a.a.O. (Fn. 11), Frage QC10.

36 Beate Hoecker / Gesine Fuchs, a.a.O. (Fn. 9), S. 292. Für die nicht zu den Transformationsstaaten gehörenden neuen EU-Länder Malta und Zypern sind traditionelle Geschlechterrollenbilder gleichfalls typisch. 


\subsection{Sozialstrukturelle Faktoren}

$\mathrm{Zu}$ den weiteren Einflussgrößen der politischen Partizipation und Repräsentation zählen individuelle Voraussetzungen aus dem sozialstrukturellen Bereich, und zwar insbesondere Bildung und Berufstätigkeit sowie die persönliche Lebenssituation. Wie Untersuchungen für die europäische Ebene belegen, verringern sich mit steigendem Bildungsniveau die Unterschiede in den Interessen von Männern und Frauen an institutionalisierter Politik merklich; gleichzeitig erhöht sich auch die subjektiv wahrgenommene politische Kompetenz. ${ }^{37}$ Hatten Frauen in vielen Ländern Europas bis vor wenigen Jahren noch deutlich schlechtere Bildungschancen als Männer, so trifft das heute nicht mehr zu: „Mittlerweile haben die Frauen im allgemeinbildenden Schulwesen und in den Hochschulen fast aller EU-Länder die Männer eingeholt und weithin sogar überholt. "38 Defizite im Bildungsstand können somit kaum noch verantwortlich sein für die Unterrepräsentation von Frauen in der Politik.

Berücksichtigt man auch die Variable Berufstätigkeit, bestätigt sich für die europäischen Staaten gleichfalls der bekannte Sachverhalt, dass durch Erwerbstätigkeit das Interesse an Politik bei Männern wie Frauen zunimmt. Aber auch die parlamentarische Repräsentation von Frauen steht in einem positiven Zusammenhang zum Ausmaß der Frauenerwerbstätigkeit. Die beim Frauenanteil unter den Abgeordneten führenden EU-Staaten weisen überwiegend auch eine hohe weibliche Beschäftigungsquote auf (Ausnahmen: Belgien und Spanien). Für die Länder mit einer mittleren beziehungsweise niedrigen Repräsentation von Frauen in den Parlamenten ist dagegen in der Regel auch nur eine mittlere/niedrige Frauenbeschäftigungsquote typisch (vgl. Tabelle 5).

Darüber hinaus setzen außerfamiliäre Aktivitäten von Frauen soziale Einrichtungen voraus, die ihnen ein berufliches wie politisches Engagement überhaupt ermöglichen. Während insbesondere die nordischen Staaten ein entsprechendes Angebot weitgehend geschaffen haben, besteht in vielen anderen europäischen Ländern (und nicht zuletzt in Deutschland) hier noch erheblicher Nachholbedarf.

\section{Fazit: Zusammenhang zwischen institutionellen, sozialstrukturellen und kulturellen Faktoren noch ungeklärt}

Für den unterschiedlichen Stand der parlamentarischen Repräsentation von Frauen in Europa gibt es keine monokausale Erklärung. Entscheidend ist vielmehr der jeweilige nationale „Cocktail“ der verschiedenen Einflussfaktoren. Zusammenfassend lassen sich aber zumindest folgende Tendenzen erkennen:

- Hohe parlamentarische Repräsentation von Frauen geht einher mit einer frühen/„mittleren“ Einführung des Frauenwahlrechts, die zudem teilweise zeitgleich mit dem Männerwahlrecht erfolgte; mit einer eher egalitären Politischen Kultur; mit einem Verhältniswahlrecht und (überwiegend) Quoten für öffentliche Kandidaturen sowie mit einer in der Regel hohen Frauenerwerbsquote.

37 Vgl. ebenda, S. 14.

38 Stefan Hradil, Sozialstruktur und gesellschaftlicher Wandel, in: Oscar W. Gabriel / Sabine Kropp (Hrsg.), a.a.O. (Fn. 7), S. 89 - 123, S. 108. 
- Typisch für mittlere parlamentarische Repräsentation von Frauen ist eine gleichfalls „mittlere“ Einführung des Frauenwahlrechts; ein eher traditionelles Geschlechterrollenbild; ein Verhältniswahlsystem und Quoten sowie eine hohe oder mittlere Frauenerwerbstätigkeit.

- Charakteristisch für niedrige parlamentarische Repräsentation von Frauen ist eine überwiegend späte Einführung des Frauenwahlrechts; ein ausgeprägt traditionelles Geschlechterrollenbild; ein Mischwahlsystem und überwiegend Quoten für öffentliche Kandidaturen.

Diese Generalisierungen sind zweifellos noch wenig befriedigend, und es bedarf weiterer Forschungen, die insbesondere die Zusammenhänge zwischen institutionellen, sozialstrukturellen und kulturellen Faktoren in ihrer Auswirkung auf die politische Repräsentation von Frauen genauer erfassen. Auf der politischen Handlungsebene sind zudem insbesondere die nationalen Regierungen gefordert, die in der Europäischen Verfassung verankerte Gleichberechtigung von Frauen und Männern auch tatsächlich umzusetzen. Eine starke Unterstützung durch zivilgesellschaftliche Organisationen wäre dabei sicherlich hilfreich. Fest steht: Die Demokratisierung der Geschlechterverhältnisse in Politik wie Gesellschaft bleibt auch zu Beginn des 21. Jahrhunderts auf der politischen Agenda.

\title{
Parlamentarierinnen in der islamischen Welt - untergeordnete Abgeordnete?
}

\author{
Claudia Derichs, Hala Kindelberger und Christine Holike
}

Parlamente in Staaten mit muslimischer Bevölkerungsmehrheit sind seltener Gegenstand der Forschung als die Exekutiven solcher Länder. ${ }^{1}$ Dies liegt daran, dass Parlamenten oder Beratenden Versammlungen, wie sie dort häufig heißen, nur eine schwache Position im politischen Entscheidungsgefüge attestiert wird, sie nur zu einem Teil aus gewählten Mitgliedern bestehen und meist keine gesetzgebende, sondern allenfalls eine entwurfsdiskutierende Funktion einnehmen. Allzu häufig dienen sie eher der Regimestabilisierung denn dem politischen Wettbewerb. ${ }^{2}$ Noch schwächer als der Einfluss der Parlamente selbst wird in aller Regel der Einfluss beurteilt, den weibliche Abgeordnete auf das parlamentarische Geschehen ausüben. Diese weit verbreitete Einschätzung soll nachfolgend aus einer differenzierten Perspektive untersucht werden. Auf der empirischen Ebene werden diejenigen geographischen Regionen betrachtet, deren politische, wirtschaftliche und gesellschaftliche Entwicklung eine äußerst intensive Ausstrahlung auf „die islamische Welt“ ausüben und die über gewählte parlamentarische Institutionen verfügen. Es handelt sich dabei um die Regionen des Persischen Golfs und Nordafrikas - untersucht werden drei Kernländer des

1 Die Exekutiven untersuchen Andrea Fleschenberg / Claudia Derichs, Handbuch Spitzenpolitikerinnen, Wiesbaden 2008.

2 Vgl. Marsha Pripstein Posusney, Multiparty Elections in the Arab World: Election Rules and Opposition Responses, in: dies. / Michele Penner Angrist (Hrsg.), Authoritarianism in the Middle East. Regimes and Resistance, Boulder 2005, S. $91-118$. 